\title{
ULTIMATE THEORETICAL STRENGTH OF FCC Fe-Ni ALLOY POLYCRYSTALS
}

\author{
S. A. Starikov, A. R. Kuznetsov*, L. E. Karkina, V. V. Sagaradze \\ M.N. Miheev Institute of Metal Physics of Ural Branch of Russian Academy of Sciences, 18 S. Kovalevskoy st., \\ Ekaterinburg, Russian Federation \\ *Corresponding author. E-mail: a kuznetsov@imp.uran.ru; address for correspondence: 18, ul. S. Kovalevskoy, 620990
Ekaterinburg, Russian Federation; phone: +7 (343) 37442 14; fax: +7 (343) 3745244 \\ An atomistic analysis of the fracture in fcc iron and the Fe-30 at. \% Ni alloy (for an ideal lat- \\ tice and near the special grain boundary) has been performed with the application of the molecular \\ dynamics method. To characterize the fracture energy, the decohesion energy and Griffith surface \\ energy for the crack planes have been calculated. A slight difference has been obtained in the ten- \\ dency of Fe to fracture along the body and along the grain boundary. The Fe-30 at. \% Ni alloy is \\ more prone to fracture along the grain boundary. The energy of interaction between $\mathrm{Ni}$ and the \\ grain boundary has been calculated, and it is shown that $\mathrm{Ni}$ atoms in the Fe-30 at. \% Ni alloy do not \\ have their own thermodynamic impetuses for segregation on the special boundary.
}

Keywords: intergranular fracture, atomistic modeling, grain boundary, energy of decohesion, segregation.

DOI: $10.17804 / 2410-9908.2015 .6 .058-062$

\section{References}

1. Bitzek E., Kermode J.R., Gumbsch P. Atomistic aspects of fracture. International Journal of Fracture, 2015, vol. 191, iss. 1, pp. 13-30. DOI: 10.1007/s10704-015-9988-2.

2. Terentyev D., He X. Properties of grain boundaries in BCC iron and iron-based alloys. An atomistic study. In: Open Report of the Belgian Nuclear Research Centre. SCK •CEN-BLG-1072, 2010, 70 p. ISSN 1379-2407.

3. [XMD] Sait paketa programm [Software Pack Site]. Available at: http://xmd.sourceforge.net/about.html. (In Russian).

4. Meyer R., Entel P. Martensite-austenite transition and phonon dispersion curves of $\mathrm{Fe}_{1-\mathrm{x}} \mathrm{Ni}_{\mathrm{x}}$ studied by molecular-dynamics simulations. Physical Review B, 1998, vol. 57, iss. 9, pp. 5140-5143. DOI: 10.1103/PhysRevB.57.5140.

5. $\quad$ Chen S.P., Voter A.F., Albers K.C., Boring A.M., Hay P.J. Investigation of the effects of boron on $\mathrm{Ni}_{3} \mathrm{Al}$ grain boundaries by atomistic simulations. Journal of Material Research, 1990, vol. 5, iss. 05, pp. 955-970. DOI: 10.1557/JMR.1990.0955.

6. Gornostyrev Yu.N, Katsnelson M.I., Medvedeva N.I., Mryasov O.N., Freeman A.J., Trefilov A.V. Peculiarities of defect structure and mechanical properties of iridium: Results of $a b$ initio electronic structure calculations. Physical Review B, 2000, vol. 62, iss 12, pp. 7802-7808. DOI: 10.1103/PhysRevB.62.7802 
Подана в журнал:30.10.2015

УДК 669.14:539.4

DOI: $10.17804 / 2410-9908.2015 .6 .058-062$

\title{
ПРЕДЕЛЬНАЯ ТЕОРЕТИЧЕСКАЯ ПРОЧНОСТЬ ПОЛИКРИСТАЛЛОВ ГЦК СПЛАВОВ Fe-Ni
}

\author{
С. А. Стариков, А. Р. Кузнецов*, Л. Е. Карькина, В. В. Сагарадзе \\ Федеральное государственное бюджетное учреждение науки Институт физики металлов имени \\ М.Н. Михеева Уральского отделения Российской академии наук, ул. С.Ковалевской, 18, 620990, \\ Екатеринбург, Российская Федераџия
}

\begin{abstract}
*Ответственный автор. Электронная почта: a_kuznetsov@imp.uran.ru; адрес для переписки: ул. С. Ковалевской, 18, 620990, Екатеринбург, Российская Федерация. Телефон: +7 (343) 374-42-14; Факс: +7 (343) 374-52-44
\end{abstract}

Проведен атомистический анализ разрушения в ГЦК железе и сплаве $\mathrm{Fe}-30$ ат. \% Ni (как для идеальной решетки, так и вблизи специальной границы зерна) с использованием метода молекулярной динамики. Для характеристики разрушения рассчитана энергия декогезии и поверхностная энергия Гриффитса для плоскостей раскрытия трещин. Получено небольшое различие в склонности $\mathrm{Fe}$ к разрушению по телу и по границе зерна. Сплав $\mathrm{Fe}-30$ aт. \% Ni скорее склонен к разрушению по границе зерна. Рассчитана энергия взаимодействия Ni с границей зерна, и показано, что атомы Ni в сплаве $\mathrm{Fe}-30$ ат. \% Ni не имеют собственных термодинамических стимулов для сегрегации на специальной границе.

Ключевые слова: межкристаллитное разрушение, атомистическое моделирование, граница зерна, энергия декогезии, сегрегащии.

\section{1. Введение}

Способность трещины к нормальному раскрытию характеризуется значением поверхностной энергии Гриффитса. Очевидно, что макроскопические процессы разрушения практически полностью определяются процессами, происходящими на атомном уровне [1]. В то время как моделированию разрушения в ОЦК-железе и сплавах на его основе уделялось большое внимание в последнее время $[1,2]$, ГЦК-железо и сплавы на его основе в этом плане менее изучены на атомном уровне. Следует отметить, что при изучении разрушения по границе зерна (ГЗ) в сплаве необходимо учитывать возможность сегрегации компонентов на ГЗ. В работе на атомном уровне рассмотрены процессы разрушения в ГЦК-железе, в сплаве $\mathrm{Fe}-$ 30 ат. \% Ni и влияние ГЗ с учетом возможности сегрегации никеля на ГЗ.

\section{2. Постановка задачи и методика моделирования}

При анализе процессов разрушения и сегрегации на ГЗ применен метод молекулярной динамики (МД), использован известный пакет программ XMD, разработанный Центре компьютерного моделирования Университета Коннектикута (США) [3]. Использовались ЕАМпотенциалы [4], хорошо апробированные для описания когезионных характеристик и полиморфного превращения в системе Fe-Ni. Моделирование проводилось для достаточно большого поликристалла, содержащего несколько зерен с границами наклона различного типа, в работе рассмотрена несимметричная специальная граница $\Sigma 5[001]\{710\} /\{110\}$ (ось разориентации [001], угол разориентации $\left.\Theta=53,13^{\circ}\right)$. На рис. 1 изображен кристаллит с тройным стыком, а также кристаллит с изучаемой границей зерна $(\Sigma 5)$.

С целью определить термодинамические стимулы для образования сегрегаций на ГЗ провели расчет энергии взаимодействия Ni с ГЗ в ГЦК-сплаве Fe-30aт. \% Ni. Поскольку в сплаве энергия легирующего элемента зависит от локального окружения, энергия его взаимодействия с границей вычислялась путем усреднения по набору конфигураций (атом Ni за- 
open-recess journal

мещал атом $\mathrm{Fe}$ на заданном расстоянии от $Г 3$, и считалась энергия кристаллита; такой расчет повторялся десятки раз для данного расстояния от ГЗ, а затем энергия усреднялась), в которых наблюдаемый атом Ni находился на заданном расстоянии от границы.

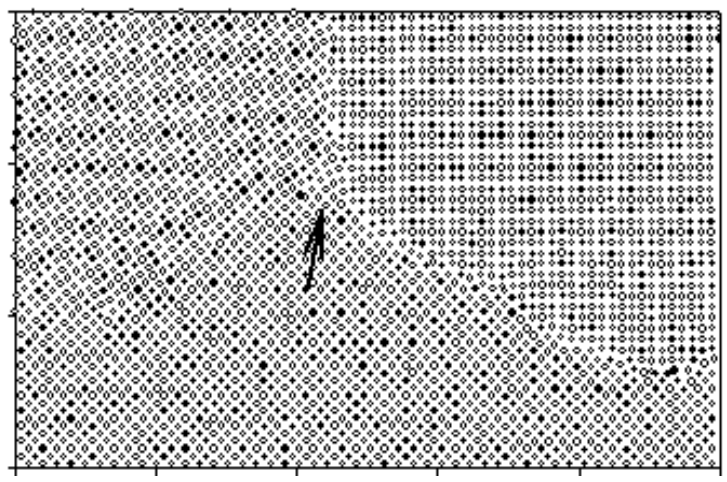

$a$

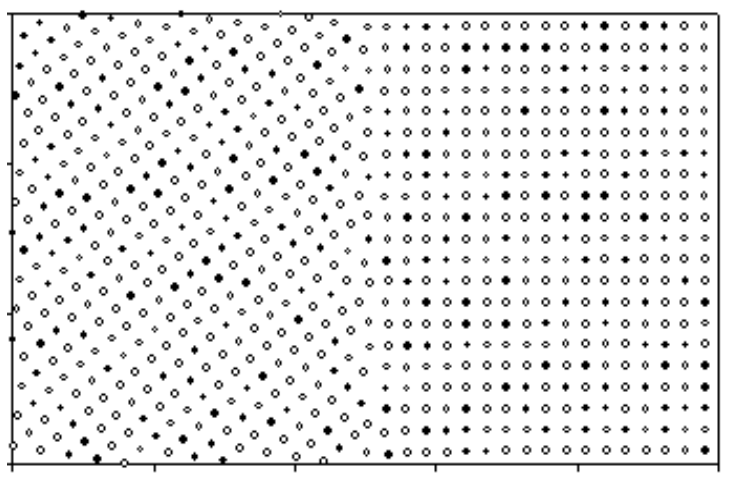

$\sigma$

Рис. 1. Кристаллит сплава Fe-30 ат. \% Ni (темные кружки - Ni) с тройным стыком (a) (обозначен стрелкой) и с ГЗ $\Sigma 5[001]\{710\} /\{110\}$ (б)

Энергию декогезии как функцию расстояния между атомными плоскостями вычисляли для одной из экспериментально наблюдаемых плоскостей скола $\{100\}$ как в кристаллите без дефектов, так и вблизи ГЗ.

\section{3. Результаты и обсуждение}

На рис. 2 представлена зависимость разности средних энергий $\mathrm{E}-\mathrm{E}_{0}$, где $\mathrm{E}-$ средняя по конфигурациям энергия кристаллита, в котором наблюдаемый атом $\mathrm{Ni}$ в сплаве $\mathrm{Fe}-30$ ат. \% Ni находился на заданном расстоянии; $\mathrm{E}_{0}-$ средняя по конфигурациям энергия кристаллита, в котором наблюдаемый атом Ni находился вдали от ГЗ; от расстояния X до ГЗ (указаны доверительные интервалы), что соответствует средней энергии взаимодействия Ni c ГЗ. $\mathrm{X}=0$ соответствует точке, расположенной в центре ГЗ.

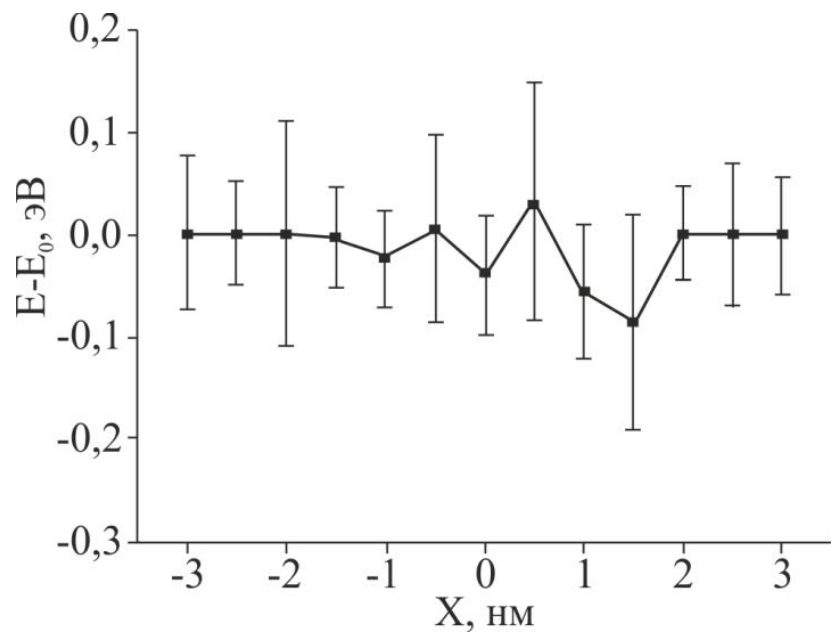

Рис. 2. Средняя энергия взаимодействия Ni с ГЗ $\Sigma 5[001]\{710\} /\{110\}$

Из рис. 2 следует, что атомы никеля довольно слабо взаимодействуют с данной специальной ГЗ (энергия сегрегации менее $0,1 \mathrm{eV}$ ), поскольку имеют близкие к атомам матрицы ионный радиус и электронную структуру. Таким образом, атомы никеля в сплаве $\mathrm{Fe}-30$ ат. \% Ni не имеют собственных термодинамических стимулов для сегрегации на ГЗ. Это обстоятельство дает возможность изучать разрушение по ГЗ в сплаве $\mathrm{Fe}-30$ aт. \% Ni без учета сегрегации на ГЗ. 
На рис. 3 a сопоставлены значения выигрыша поверхностной энергии G кристаллита для чистого Fe с ГЦК-решеткой (кривая 1) и неупорядоченного сплава Fe-30 ат. \% $\mathrm{Ni}$ (кривая 2). В табл. 1 даны значения энергии декогезии $\mathrm{G}_{\mathrm{c}}$ и поверхностной энергии Гриффитса $\gamma_{\mathrm{C}}=\frac{G_{C}}{2}$. Энергия Гриффитса вычисляется как разность поверхностной энергии кристаллита и энергии $\Gamma 3[5]: \gamma_{C}=\frac{\left(G-\gamma_{\Gamma 3}\right)}{2}$ (для идеальной решетки $\left.\gamma_{\Gamma 3}=0\right)$. Видно, что в идеальной решетке легирование Ni увеличивает значение поверхностной энергии, что затрудняет процесс раскрытия трещины (табл. 1).

На рис. 3 б и в табл. 1 приведены вычисленные значения энергии декогезии и энергии поверхностных дефектов вблизи несимметричной границы зерна $\Sigma 5[001]\{710\} /\{110\}$. Плоскость ГЗ $\{710\}$ приблизительно на $8^{\circ}$ отличается от плоскости $\{100\}$. Из сопоставления данных в табл. 1 следует, что значения энергии декогезии вблизи ГЗ ниже, чем в идеальной решетке. Влияние легирования при разрушении по ГЗ противоположно случаю идеальной решетки. Легирование уменьшает значение энергии поверхностных дефектов, что свидетельствует о том, что процесс раскрытия трещины в этом случае осуществляется легче.

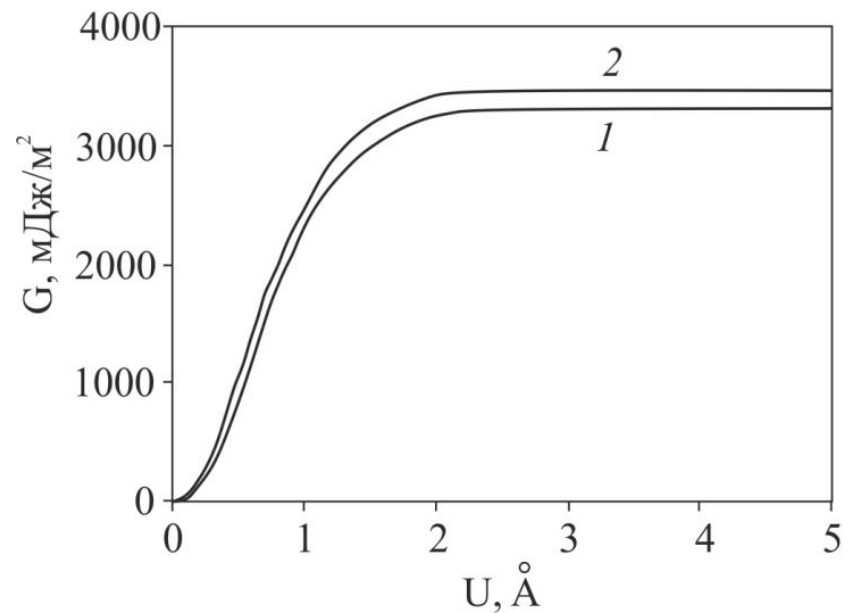

$a$

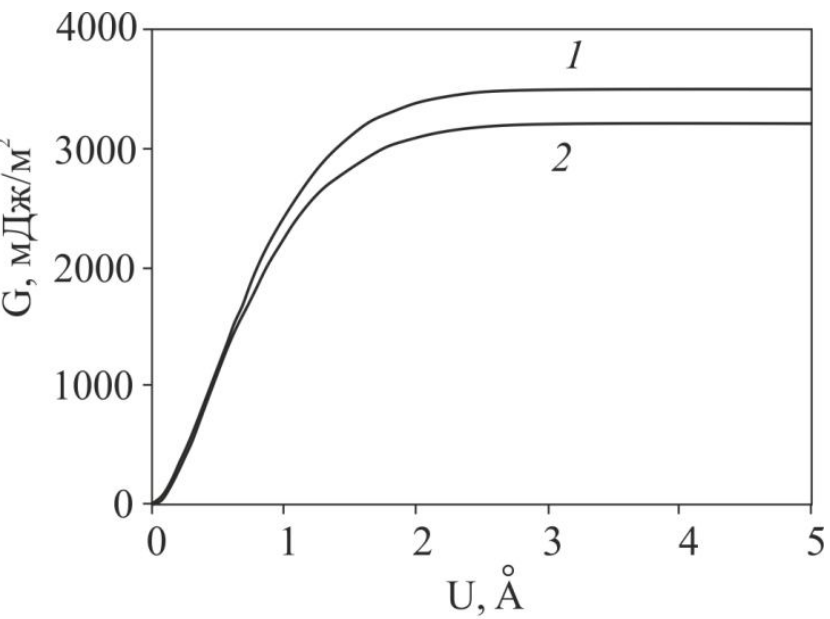

$\sigma$

Рис. 3. Изменение энергии кристаллита $\mathrm{G}$ в зависимости от расстояния между атомными плоскостями $\{100\}: a$ - для идеальной решетки; $\sigma$ - для плоскости по ГЗ. Кривая $1-$ ГЦК-Fe; кривая 2 - Fe-30 ат. \% Ni

Таблица 1 - Энергия декогезии $\mathrm{G}_{\mathrm{c}}$ и поверхностная энергия Гриффитса $\gamma_{\mathrm{c}}=\mathrm{G}_{\mathrm{c}} / 2$ для плоскостей $\{100\}$ раскрытия трещин в $\mathrm{Fe}$ и $\mathrm{Fe}-30$ ат. \% Ni

\begin{tabular}{|c|c|l|l|}
\hline Материал & Объект & $\mathrm{G}_{\mathrm{c}}, \mathrm{MДж/ \textrm {m } ^ { 2 }}$ & $\gamma_{\mathrm{c}}, \mathrm{мДж/ \textrm {m } ^ { 2 }}$ \\
\hline $\mathrm{Fe}$ & $\begin{array}{c}\text { Идеальная ГЦК } \\
\text { решетка }\end{array}$ & 3304 & 1652 \\
\hline $\mathrm{Fe}-30 \mathrm{aт.} \% \mathrm{Ni}$ & $-/ /-$ & 3456 & 1728 \\
\hline $\mathrm{Fe}$ & Для Г3 & 3238 & 1619 \\
\hline $\mathrm{Fe}-30 \mathrm{aт.} \% \mathrm{Ni}$ & $-/ /-$ & 2618 & 1309 \\
\hline
\end{tabular}

Для хорошо деформируемых чистых металлов экспериментальные значения поверхностной энергии $\gamma_{\mathrm{c}} \approx 1200-2300$ мДж/м² [6].

\section{4. Заключение}

Таким образом, как $\mathrm{Fe}$, так и сплав $\mathrm{Fe}-30$ ат. \% Ni, относятся к хорошо деформируемым металлам, обладающим сравнительно высоким значением поверхностной энергии 
Гриффитса. Значения поверхностной энергии Гриффится в целом выше для транскристаллитного разрушения по сравнению с межкристаллитным разрушением. Можно отметить небольшое различие в склонности Fе к разрушению как по телу, так и по границе зерна. Сплав Fe-30 ат. \% Ni скорее разрушается по ГЗ (табл. 1). Атомы Ni в сплаве Fe-30 ат. \% Ni не имеют собственных термодинамических стимулов для сегрегации на ГЗ.

\section{Благодарность}

Работа выполнена в рамках государственного задания ФАНО России по теме «Структура» ИФМ УрО РАН. Авторы благодарны Ю.Н. Горностыреву за полезное обсуждение.

\section{Литература}

1. Bitzek E., Kermode J. R., Gumbsch P. Atomistic aspects of fracture // International Journal of Fracture. - 2015. - Vol. 191, iss. 1. - P. 13-30. - DOI: 10.1007/s10704-015-9988-2.

2. Terentyev D., He X. Properties of grain boundaries in BCC iron and iron-based alloys. An atomistic study // Open Report of the Belgian Nuclear Research Centre. - SCK •CEN-BLG-1072 Belgium, 2010. - 70 p. - ISSN 1379-2407.

3. XMD [Электронный pecypc] // Сайт пакета программ. URL: http://xmd.sourceforge.net/about.html.

4. Meyer R., Entel P. Martensite-austenite transition and phonon dispersion curves of $\mathrm{Fe}_{1-\mathrm{x}} \mathrm{Ni}_{\mathrm{x}}$ studied by molecular-dynamics simulations // Physical Review B. - 1998. - Vol. 57, iss 3. - P. 5140-5143. - DOI 10.1103/PhysRevB.57.5140.

5. Chen S. P., Voter A. F., Albers K. C., Boring A. M., Hay P. J. Investigation of the effects of boron on $\mathrm{Ni}_{3} \mathrm{Al}$ grain boundaries by atomistic simulations // Journal of Material Research. - 1990. Vol. 5, iss. 05. - P. 955-970. - DOI: 10.1557/JMR.1990.0955.

6. Gornostyrev Yu. N, Katsnelson M. I., Medvedeva N. I., Mryasov O. N., Freeman A. J., Trefilov A. V. Peculiarities of defect structure and mechanical properties of iridium: Results of $a b$ initio electronic structure calculations // Physical Review B. - 2000. - Vol. 62. - P. 7802-7808. DOI 10.1103/PhysRevB.62.7802. 